\title{
Análise dos trabalhos de conclusão de curso da graduação em enfermagem da UNI MONTES
}

\section{Analysis of the work of completion of the graduate course in nursing's UNI MONTES}

\section{Análisis de la obra de finalización del curso de postgrado en enfermería del UNI MONTES}

\author{
Valdete da Silva', Ana Paula Ferreira Holzmann", Clara de Cássia Versiani"l', Maria Fernanda Santos
}

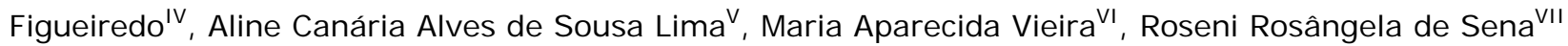

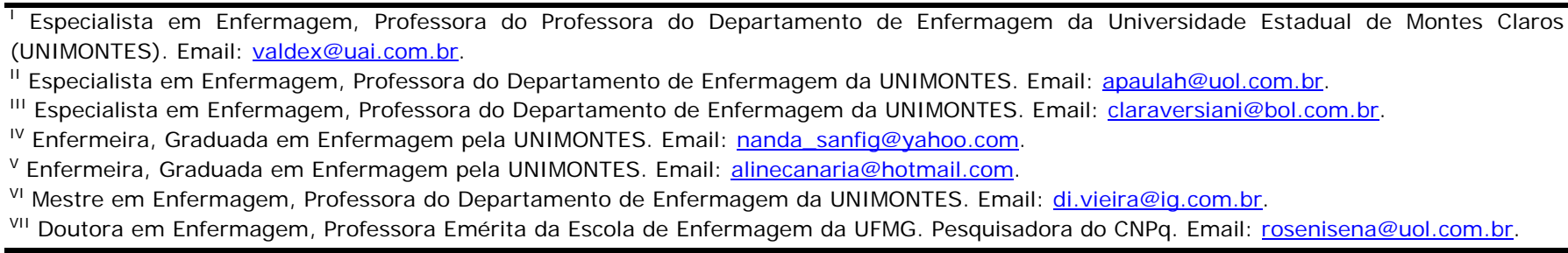

\section{RESUMO}

O Trabalho de Conclusão de Curso constitui-se em requisito obrigatório e indispensável à integralização do curso, sendo instrumento importante de avaliação de influências e tendências em Cursos de Enfermagem. É adotado na forma de monografia pela Graduação em Enfermagem da Universidade Estadual de Montes Claros (UNIMONTES), em Minas Gerais. O objetivo deste estudo foi identificar as tendências temáticas e metodológicas das monografias dos acadêmicos desse curso. Trata-se de estudo documental e descritivo, realizado no período de janeiro a junho de 2007 , na Biblioteca Central da UNIMONTES. Os dados foram coletados em 263 monografias por meio de formulário para posterior agrupamento por áreas contempladas; metodologia; tipos de estudo; instrumentos; sujeitos do estudo e cenários. Os resultados indicaram que a área assistencial foi escolhida pela maioria dos graduandos, sendo a Saúde da Mulher a temática mais estudada. A metodologia preconizada foi qualitativa e os estudos descritivos. Os Hospitais e a Estratégia Saúde da Família foram opções significativas como cenários e o instrumento de coleta de dados mais utilizado foi a entrevista. Espera-se que esses resultados possam contribuir para a definição de diretrizes e estratégias a fim de adequar, ampliar e consolidar as atividades de pesquisa como ferramenta indispensável no Curso de Enfermagem.

Descritores: Monografia; Enfermagem; Pesquisa.

\section{ABSTRACT}

The monograph is an essential requirement to the integration of the course, being important tool for evaluation of influences and trends in nursing courses. It is adopted by undergraduate nursing at the State University of Montes Claros (UNIMONTES), in Minas Gerais/Brazil. The purpose of this study was to identify trends and methodological issues of monographs of students on this course. It is documentary and descriptive study, carried out from January to J une 2007, the Central Library UNIMONTES. Data were collected in 263 monographs using a form to group the areas; methodology; types of study; instruments; subject of study and scenarios. The results indicated that the area care was chosen by the majority of graduate students, and the Women's Health the most studied theme. The most studied methodology was qualitative and descriptive. Hospitals and the Family Health Strategy were significant options as scenarios and as instrument data collection the interview was used. It is hoped that these results can contribute to the development of guidelines and strategies to adapt, expand and consolidate the research activities as indispensable tool in the Course of Nursing.

Descriptors: Monograph; Nursing; Research.

\section{RESUMEN}

La monografía es un requisito esencial para la integración de curso, siendo importante herramienta para la evaluación de las influencias y tendencias en los cursos de enfermería. Es adoptada por el Curso de Enfermería en la Universidad Estatal de Montes Claros (UNIMONTES), en Minas Gerais. El objetivo de este estudio fue identificar las tendencias y las cuestiones metodológicas de monografías de los estudiantes en este curso. Se trata de estudio documental y descriptivo, realizado entre enero y junio de 2007, en la Biblioteca Central UNIMONTES. Los datos fueron recolectados en 263 monografías utilizando formulario para puesto en grupo áreas: metodología; tipos de estudio; instrumentos objeto de estudio y escenarios. Los resultados indicaron que el área de atención fue elegido por la mayoría de los estudiantes de posgrado, y la Salud de la Mujer el tema más estudiado. La metodología más estudiada fue cualitativa y descriptiva. Los hospitales y en la Estrategia Salud de la Familia fueron importantes opciones como escenarios y como instrumento de recopilación de datos se utilizó la entrevista. Es de esperar que estos resultados pueden contribuir a la elaboración de directrices y estrategias para adaptar, ampliar y consolidar las actividades de investigación como herramienta indispensable en el Curso de Enfermería.

Descriptors: Monografía; Enfermería; Investigación. 


\section{NTRODUÇÃO}

A pesquisa é ferramenta importante e indispensável no processo de trabalho do enfermeiro. Integra as competências a serem desenvolvidas durante a graduação e deve tornar-se a base da prática profissional vinculada à realidade local e ao contexto histórico em que é realizada ${ }^{(1)}$.

Constitui-se como estratégia fundamental para a construção do conhecimento, sendo que as teorias e o conhecimento gerados a partir de pesquisa em Enfermagem são essenciais para o estabelecimento de uma base científica que garanta a qualidade do cuidado e a credibilidade profissional $^{(1)}$. Além disso, retrata traços do perfil acadêmico, sendo também um instrumento importante de avaliação das influências e tendências de Cursos de Enfermagem ${ }^{(1-2)}$.

Para tanto, a formação de pesquisadores deve ter início na graduação, visto que é nessa etapa também que se deve apresentar e valorizar a cultura do consumo e produção de pesquisa. Esforços para capacitação de quem se inicia no caminho da pesquisa são necessários para garantir o crescimento, a qualidade, a continuidade e a valorização da produção de conhecimento na área ${ }^{(1)}$.

É com a pesquisa que o estudante aprende a utilizar a metodologia científica para detectar, conhecer, resolver situações e propor ações que necessitam de intervenção; incentivando o estudante a prosseguir em sua formação acadêmica. Entretanto, algumas vezes, a educação profissional coíbe a criatividade e a individualidade dos estudantes, por oferecer-lhes, como alternativa formal, a aquisição passiva de conhecimentos, pois o processo de formação e capacitação de recursos humanos necessita estar ligado ao desenvolvimento da criticidade do educando para a habilitação de um profissional ativo e capaz de articular seus pensamentos e idéias ${ }^{(3)}$.

O Trabalho de Conclusão de Curso (TCC) é uma exigência para a conclusão dos cursos superiores no País, constituindo-se em requisito obrigatório. Tratase de uma atividade indispensável à integralização do Curso, utilizada como indicador na avaliação da qualidade institucional e, ainda, enquanto contribuição social ${ }^{(2)}$.

Compreende a elaboração individual de um trabalho, sob orientação docente. Devem ser observadas as exigências metodológicas e os requisitos técnicos pertinentes à apresentação de trabalhos científicos, obedecendo às normas da Associação Brasileira de Normas Técnicas (ABNT).

O Curso de Graduação em Enfermagem da Universidade Estadual de Montes Claros (UNIMONTES) em Minas Gerais, em concordância com as Diretrizes Curriculares Nacionais para a área da Enfermagem, definiu no seu Projeto Pedagógico de Curso (PPC), em 2001, o TCC como parte integrante e obrigatória de suas atividades curriculares, utilizando como modalidade a Monografia.

No processo de consolidação do Curso de Enfermagem da UNIMONTES, a sistematização do conhecimento e a procura de novos campos a serem desenvolvidos, ampliando as abordagens metodológicas e de correntes de pensamento, favoreceram a criação do Grupo de Pesquisa em Enfermagem em 2005. O Grupo criou as condições para que a pesquisa seja incorporada à prática dos docentes e dos estudantes, considerando que a Enfermagem deve atuar na assistência, no ensino, na pesquisa e na gestão, tomados, cada um, em sua amplitude. O Grupo desenvolve duas linhas de pesquisa: Epidemiologia e Cuidado em Enfermagem humanização com enfoque de gênero.

Objetivando adequar a produção acadêmica advinda da elaboração do TCC às linhas de pesquisas, formulou-se este estudo, cujo objetivo foi identificar as tendências temáticas e metodológicas dos Trabalhos de Conclusão de Curso dos acadêmicos da Graduação em Enfermagem da UNI MONTES.

Em relação a temática abordada nesta investigação há vários estudos como o realizado no Curso de Enfermagem da Universidade Estadual do Paraná, em 2004, que trata das diferentes abordagens dos TCC. Observou-se que entre os trabalhos analisados nesta pesquisa, 143 (59,2\%) foram da área da Saúde Coletiva e 35 (14,6\%) da Saúde da Mulher ${ }^{(4)}$. Provavelmente o desenvolvimento das pesquisas, com destaque para a área de Saúde Coletiva, ocorreu por estarem de acordo com o perfil proposto para os egressos do curso da citada Universidade ${ }^{(4)}$.

Em um estudo realizado sobre o perfil da produção científica da graduação em enfermagem apresentada no Simpósio Internacional de Iniciação Científica da Universidade de São Paulo (SIICUSP), entre os anos de 1997 a 2004, verificou-se que entre as temáticas estudadas nesta pesquisa relacionada com os serviços de saúde apareceram em apenas $0,3 \%$ dos trabalhos analisados no período, demonstrando um provável desinteresse dos estudantes por um assunto de grande importância para proporcionar ao graduando uma formação adequada $^{(1)}$.

Quanto às metodologias utilizadas na elaboração do TCC - 81,8\% dos estudos analisados no Curso de Graduação em Enfermagem do Paraná foram qualitativos $^{(4)}$.

$\mathrm{Na}$ área da Enfermagem, as ações de intervenção devem ser norteadas pelas necessidades apontadas pelos usuários e/ou clientes e não só definidas sob o ponto de vista do prestador da assistência, o que é possível de se realizar pela pesquisa em Enfermagem, pois permite construir 
uma base de conhecimento adequado à implementação de seus resultados cientificamente validados na prática, além de fortalecer a profissão ${ }^{(5)}$. Estas afirmações resultaram de estudos realizados no Curso de Graduação da Escola de Enfermagem da Universidade de São Paulo, em 2004, sobre estratégias de ensino de pesquisa qualitativa.

Acredita-se que a tendência à abordagem qualitativa seja um reflexo do desenvolvimento galgado pela Enfermagem como profissão desde a modernidade com Florence, voltando-se muito mais para influências filosóficas como o humanismo e a fenomenologia, e muito menos para o positivismo que foi se mostrando inadequado à busca de compreensão e explicação dos fenômenos sociais, fazendo emergir novas abordagens metodológicas nas investigações científicas ${ }^{(6)}$. Ressalta-se também a necessidade de que o enfermeiro tenha uma visão holística, destacando a subjetividade e a singularidade do usuário, bem como a apreensão de termos como vínculo, acolhimento, afetividade e respeito para superar as conseqüências de um ensino fragmentado e reducionista ${ }^{(7)}$.

Estudos sobre temáticas qualitativas são de fundamental importância, considerando que o grande compromisso e desafio de quem gerencia o cuidado é o de utilizar as relações enquanto tecnologia, para buscar uma construção mútua entre os sujeitos, dando sustentação à satisfação das necessidades dos indivíduos, valorizando trabalhadores e usuários como potentes para intervirem na concretização do cuidado $^{(8)}$.

Entretanto, o Ministério da Saúde ${ }^{(9)}$ ressalta que o espectro da pesquisa em Saúde é amplo e deve incluir pesquisa biomédica; pesquisa em saúde pública; pesquisa em sistemas e políticas de saúde; pesquisa em saúde ambiental; pesquisa em ciências sociais e comportamentais; pesquisa operacional e pesquisa em saúde como parte da pesquisa geral em "ciência e tecnologia", necessitando, dessa forma, de uma diversificação dos sujeitos das pesquisas a serem realizadas, a fim de contemplar os diferentes campos da pesquisa em saúde para preencher os inúmeros vazios observados na produção científica.

Quanto aos tipos de estudos, verificou-se em pesquisas realizadas sobre o perfil da produção científica da graduação em enfermagem apresentada no Simpósio Internacional de Iniciação Científica da Universidade de São Paulo (SIICUSP), entre os anos de 1997 a 2004, que a produção de estudos foram do tipo não experimental, correspondente a $98,1 \%$ do total de estudos apresentados no período ${ }^{(1)}$. Algumas possíveis explicações para esse dado poderiam estar associadas a exigência de uma alta produtividade em pesquisa e um outro aspecto que pode ser considerado como relevante para o predomínio da pesquisa não-experimental na Enfermagem consiste no processo de formação de pesquisadores enfermeiros que não privilegia a pesquisa básica nem a experimental ${ }^{(1)}$.

Assim sendo, os resultados desta investigação deverão contribuir para a definição de diretrizes e estratégias para ampliar e consolidar as atividades de pesquisa com a qualidade que novos compromissos demandam para o Curso de Enfermagem da UNIMONTES. Este estudo possibilita, ainda, uma reorientação para o contexto atual da pesquisa nos diversos cenários de atuação do Curso de Graduação em Enfermagem e para a Pós-graduação que ora se inicia no Departamento de Enfermagem.

Considera-se que para o crescimento da profissão é relevante conhecer quantitativos e qualitativos da produção científica em geral e das áreas de saber específicas, na procura de evidências não somente sobre o desenvolvimento profissional, mas, fundamentalmente, sobre os resultados e impactos do progresso científico e tecnológico na saúde da população ${ }^{(10)}$.

\section{METODOLOGI A}

Trata-se de um estudo documental, retrospectivo, cuja fonte de coleta de dados refere-se a documentos denominados de fonte primária. Optou-se pela abordagem quantitativa, de caráter descritivo, uma vez que os fenômenos ou fatos são observados, registrados e analisados, sem serem manipulados.

A coleta de dados ocorreu no período de janeiro a junho de 2007 na Biblioteca Central da UNIMONTES, onde estão indexadas as monografias do Curso de Enfermagem. O estudo contemplou 282 Trabalhos de Conclusão de Curso produzidos de 2001 a 2006. Destes, foram resgatados 263 trabalhos para análise, sendo que os 19 não recuperados foram excluídos do estudo.

Essas monografias foram submetidas e avaliadas por uma Banca Examinadora, quando de sua defesa no 8 período do Curso de Enfermagem. Todo o processo relativo à construção do estudo monográfico está contido no Manual de Orientações para a Elaboração do Trabalho de Conclusão do Curso de Graduação em Enfermagem da UNIMONTES, seguido pelos orientadores e orientandos na trajetória de elaboração das monografias.

O estudo foi realizado mediante pedido de autorização a chefe do Departamento de Enfermagem e a responsável pela Biblioteca, que assinaram um Termo concordando com a realização da investigação. Também as recomendações postas pela Resolução $n^{\circ} 196$ de 10 de outubro de 1996 do Ministério da Saúde foram seguidas, considerando que o Projeto desta pesquisa foi aprovado pelo Comitê de Ética em Pesquisa (CEP) da UNIMONTES, conforme Parecer Consubstanciado $n^{\circ} 233$ de 19 de 
setembro de 2005.

O registro dos dados coletados a partir das monografias encontradas foi efetuado em um formulário onde se preenchia a área contemplada da monografia, segundo categorias do conhecimento: assistencial, organizacional e profissional; a metodologia utilizada no estudo monográfico: qualitativa, quantitativa e quanti-qualitativa; os tipos de estudo: descritivo, exploratório, descritivo e exploratório, experimental e observacional; os instrumentos utilizados para a coleta de dados: entrevista, questionário, formulário e grupo focal; os sujeitos do estudo e os cenários das pesquisas realizadas. Constava ainda neste formulário as categorias: não especificado adequadamente, mal definido. Esse formulário foi previamente testado para verificar sua adequação. Após a coleta de dados foi realizado agrupamento dos dados e uma análise quantitativa dos mesmos a partir do registro dos formulários.

\section{APRESENTAÇÃO E DISCUSSÃO DOS RESULTADOS}

Os dados serão apresentados segundo as áreas contempladas nas monografias; metodologia utilizada; tipos de estudo; instrumentos utilizados; sujeitos do estudo e cenários das pesquisas por meio de tabelas contendo essas informações.

\section{Áreas contempladas}

A análise das 263 monografias possibilitou sua classificação nas seguintes categorias do conhecimento: assistencial, organizacional e profissional, em conformidade com as propostas de Linhas de Pesquisa em Enfermagem consolidadas no 11 은 Seminário Nacional de Pesquisa em Enfermagem ${ }^{(11)}$. A área assistencial abrange o processo de cuidar em enfermagem como determinantes de qualidade de vida, do processo saúde-doença e da história da enfermagem. A área organizacional compreende as políticas e práticas em Saúde, Educação e Enfermagem, Saúde e Trabalho, Gerenciamento dos Serviços e Informação/Comunicação na Enfermagem. A profissional inclui os Fundamentos do Cuidado de Enfermagem e de Saúde, Concepções TeóricoFilosóficas, Tecnologias e Ética na Saúde e Enfermagem ${ }^{(11)}$.

A Tabela 1 mostra as áreas contempladas nos estudos e a área assistencial foi escolhida por 199 $(75,7 \%)$ do total dos trabalhos realizados, seguida da área organizacional com 35 (13,3\%).

Tabela 1: Áreas do conhecimento contempladas nas monografias no Curso de Graduação em Enfermagem da Unimontes no período de 2001 a 2006. Biblioteca Central da UNI MONTES, 2007.

\begin{tabular}{|c|c|c|c|c|c|c|c|c|}
\hline \multirow{2}{*}{ Anos Áreas } & \multicolumn{2}{|c|}{ Profissional } & \multicolumn{2}{c|}{ Assistencial } & \multicolumn{2}{c|}{ Organizacional } & \multicolumn{2}{c|}{ Total } \\
\cline { 2 - 8 } & $\mathbf{n}$ & $\mathbf{\%}$ & $\mathbf{n}$ & $\mathbf{\%}$ & $\mathbf{n}$ & $\mathbf{\%}$ & $\mathbf{n}$ & $\mathbf{\%}$ \\
\hline 2001 & 1 & 2,5 & 32 & 80,0 & 7 & 17,5 & 40 & 15,2 \\
\hline 2002 & 5 & 14,7 & 25 & 73,5 & 4 & 11,8 & 34 & 12,9 \\
\hline 2003 & 8 & 20,5 & 27 & 69,2 & 4 & 10,3 & 39 & 14,8 \\
\hline 2004 & 5 & 12,5 & 30 & 75,0 & 5 & 12,5 & 40 & 15,2 \\
\hline 2005 & 4 & 7,5 & 43 & 83,0 & 5 & 9,5 & 52 & 19,8 \\
\hline 2006 & 6 & 10,3 & 42 & 72,5 & 10 & 17,2 & 58 & 22,1 \\
\hline TOTAL & $\mathbf{2 9}$ & $\mathbf{1 1 , 0}$ & $\mathbf{1 9 9}$ & $\mathbf{7 5 , 7}$ & $\mathbf{3 5}$ & $\mathbf{1 3 , 3}$ & $\mathbf{2 6 3}$ & $\mathbf{1 0 0}$ \\
\hline
\end{tabular}

A Enfermagem tem destacado a sua presença quantitativa e qualitativa nos âmbitos assistenciais, em torno do cuidado do ser doente e não da doença, não se reduzindo as lógicas médico-centradas ${ }^{(12)}$. Assim sendo, para proporcionar assistência de enfermagem efetiva e capaz de atender às reais necessidades do cliente, é importante a busca de conhecimentos específicos que se complementam com os conhecimentos das demais práticas dos profissionais de saúde, na concretude de uma prática social relevante e indispensável à sociedade brasileira. No desempenho profissional, o enfermeiro precisa, continuamente, conhecer acerca da saúde do indivíduo e das coletividades com aporte científico e fundamentado em ações independentes e/ou interdependentes de maneira autônoma ou em colaboração com outros profissionais ${ }^{(13)}$. A preocupação com a qualidade do cuidado reflete um maior interesse dos profissionais pelas pesquisas na área assistencial, como se verificou neste estudo, 75, 7\%.

Ademais, pesquisas na área assistencial devem estar comprometidas com a melhoria direta do contexto social, já que o ato de assistir/cuidar está incluído no processo de pesquisar. Dessa forma, a pesquisa é uma dimensão da prática social da Enfermagem e deve estar inter-relacionada ao assistir, ao cuidar, ao ensinar e ao gerenciar. A área assistencial oferece vasto campo para a pesquisa e faz avançar o processo de construção do conhecimento em Enfermagem, devendo ser a prática objeto de pesquisa(2). Os resultados deste estudo refletem sobre esses argumentos, demonstrando preocupação dos docentes e estudantes na formação do acadêmico em relação a 
essa temática no Curso de Enfermagem da UNI MONTES.

A partir das temáticas preferidas pelos estudantes, em suas monografias, foi realizado um agrupamento dessas em acordo com as orientações do Artigo 1ํ da Resolução $n^{\circ}$ 290/2004 do Conselho
Federal de Enfermagem, que trata das Especialidades de Enfermagem ${ }^{(14)}$. As temáticas mais contempladas neste estudo foram: Saúde da Mulher, 45 (17,1\%); Saúde do Adulto, $38(14,4 \%)$ e Saúde Coletiva 36 $(13,7 \%)$ (Tabela 2).

Tabela 2: Distribuição dos temas estudados nas monografias do Curso de Graduação em Enfermagem da UNIMONTES no período de 2001 a 2006. Biblioteca Central da UNIMONTES, 2007.

\begin{tabular}{|c|c|c|c|c|c|c|c|c|c|c|c|c|c|c|}
\hline \multirow{2}{*}{ Áreas } & $\mathbf{2 0 0 1}$ & $\mathbf{2 0 0 2}$ & $\mathbf{2 0 0 3}$ & $\mathbf{2 0 0 4}$ & $\mathbf{2 0 0 5}$ & $\mathbf{2 0 0}$ & \multicolumn{2}{c|}{ Total } \\
\hline & $\mathbf{n}$ & $\mathbf{\%}$ & $\mathbf{n}$ & $\mathbf{\%}$ & $\mathbf{N}$ & $\mathbf{\%}$ & $\mathbf{n}$ & $\mathbf{\%}$ & $\mathbf{n}$ & $\mathbf{\%}$ & $\mathbf{N}$ & $\mathbf{\%}$ & $\mathbf{n}$ & $\mathbf{\%}$ \\
\hline Administração em Enfermagem & 04 & 10 & 02 & 5,9 & 01 & 2,6 & - & - & 05 & 9,6 & - & - & 12 & 4,6 \\
\hline Doenças Transmissíveis & 02 & 05 & - & - & 04 & 10,3 & 03 & 7,5 & 01 & 1,9 & 05 & 8,6 & 15 & 5,7 \\
\hline Educação em Enfermagem & - & - & - & - & 01 & 2,6 & - & - & 01 & 1,9 & 01 & 1,7 & 03 & 1,1 \\
\hline Enfermagem Fundamental & 04 & 10 & 06 & 17,6 & 07 & 17,9 & 05 & 12,5 & 06 & 11,5 & 02 & 3,4 & 30 & 11,4 \\
\hline Saúde Coletiva & 06 & 15 & 02 & 5,9 & 04 & 10,3 & 05 & 12,5 & 07 & 13,5 & 12 & 20,7 & 36 & 13,7 \\
\hline Saúde da Criança & 06 & 15 & 06 & 17,6 & 04 & 10,3 & 03 & 7,5 & 05 & 9,6 & 09 & 15,1 & 33 & 12,5 \\
\hline Saúde da Mulher & 06 & 15 & 09 & 26,5 & 03 & 7,7 & 08 & 20 & 11 & 21,2 & 08 & 13,8 & 45 & 17,1 \\
\hline Saúde do Adolescente & 02 & 5 & 02 & 5,9 & 02 & 5,1 & 02 & 5 & 02 & 3,8 & 05 & 8,6 & 15 & 5,7 \\
\hline Saúde do Adulto & 04 & 10 & 04 & 11,8 & 08 & 20,5 & 05 & 12,5 & 08 & 15,4 & 09 & 15,1 & 38 & 14,4 \\
\hline Saúde do Idoso & 03 & 7,5 & 02 & 5,9 & - & - & 06 & 15 & 03 & 5,8 & 03 & 5,2 & 17 & 6,5 \\
\hline Saúde do Trabalhador & 01 & 2,5 & 01 & 2,9 & 03 & 7,7 & 03 & 7,5 & 01 & 1,9 & 02 & 3,4 & 11 & 4,2 \\
\hline Saúde Mental & 02 & 5 & - & - & 02 & 5,1 & - & - & 02 & 3,9 & 02 & 3,4 & 08 & 3,1 \\
\hline TOTAL & $\mathbf{4 0}$ & $\mathbf{1 0 0}$ & $\mathbf{3 4}$ & $\mathbf{1 0 0}$ & $\mathbf{3 9}$ & $\mathbf{1 0 0}$ & $\mathbf{4 0}$ & $\mathbf{1 0 0}$ & $\mathbf{5 2}$ & $\mathbf{1 0 0}$ & $\mathbf{5 8}$ & - & $\mathbf{2 6 3}$ & $\mathbf{1 0 0}$ \\
\hline & & & & & & & & & &
\end{tabular}

A diversidade de temas relacionados na Tabela 2 demonstra que são contemplados principalmente determinados conjuntos de ações propedêuticas: as ações de proteção à saúde; a promoção de saúde; a prevenção de doenças, as ações curativas e as ações de reabilitação. Observa-se a ausência de estudos com temáticas relacionadas ao ensino, à organização dos serviços, às novas modalidades de assistência como o processo de trabalho em saúde, à sistematização da assistência, à atenção e internação domiciliar, entre outras.

A produção de conhecimentos que resultam em impactos significativos para a sociedade requer competências de toda ordem: política, gerencial, técnica e teórica ${ }^{(15)}$. Sendo assim, há que se buscar uma reorientação para o atual contexto da pesquisa no Curso de Graduação em Enfermagem da UNIMONTES, objetivando contemplar objetos como outras tecnologias, novas formas de gestão do trabalho e articulações político-sociais que privilegiem o cuidar ético e humano na produção dos serviços de saúde, incorporando as modificações do mundo do trabalho, das relações sociais e dos padrões culturais que trazem implicações para a prática de Enfermagem ${ }^{(16)}$.

Ressalta-se que a informática se constitui como estratégica fonte para a pesquisa bibliográfica sobre os mais diferentes temas. A facilidade de acesso e a amplitude ilimitada de alcance dessa produção são poderosos recursos para atualização permanente do profissional $^{(17)}$.

\section{METODOLOGI A UTI LI ZADA}

Quanto à metodologia utilizada na elaboração dos TCC, há maior incidência de estudos qualitativos, perfazendo um total de $154(58,5 \%)$, enquanto os estudos quantitativos representam $93 \quad(35,4 \%)$, conforme mostra a Tabela 3.

Tabela 3: Metodologias utilizadas na elaboração das monografias do Curso Graduação em Enfermagem da UNIMONTES no período de 2001 a 2006. Biblioteca Central da UNIMONTES, 2007.

\begin{tabular}{|c|c|c|c|c|c|c|c|c|c|c|c|c|c|c|}
\hline \multirow{2}{*}{ Anos } & $\mathbf{2 0 0 1}$ & \multicolumn{2}{|c|}{$\mathbf{2 0 0 2}$} & \multicolumn{2}{c|}{$\mathbf{2 0 0 3}$} & $\mathbf{2 0 0 4}$ & $\mathbf{2 0 0 5}$ & $\mathbf{2 0 0}$ & \multicolumn{2}{c|}{ Total } \\
\cline { 2 - 14 } & $\mathbf{n}$ & $\mathbf{\%}$ & $\mathbf{n}$ & $\mathbf{\%}$ & $\mathbf{n}$ & $\mathbf{\%}$ & $\mathbf{N}$ & $\mathbf{\%}$ & $\mathbf{n}$ & $\mathbf{\%}$ & $\mathbf{n}$ & $\mathbf{\%}$ & $\mathbf{n}$ & $\mathbf{\%}$ \\
\hline Qualitativa & 12 & 30 & 14 & 41,2 & 28 & 71,8 & 20 & 50 & 44 & 84,6 & 36 & 62,1 & 154 & 58,5 \\
\hline Quantitativa & 15 & 37,5 & 20 & 58,8 & 11 & 28,2 & 19 & 47,5 & 7 & 13,5 & 21 & 36,2 & 93 & 35,4 \\
\hline Quanti-qualitativa & 13 & 32,5 & - & - & - & - & 1 & 2,5 & 1 & 1,9 & 1 & 1,7 & 16 & 6,1 \\
\hline TOTAL & $\mathbf{4 0}$ & $\mathbf{1 0 0}$ & $\mathbf{3 4}$ & $\mathbf{1 0 0}$ & $\mathbf{3 9}$ & $\mathbf{1 0 0}$ & $\mathbf{4 0}$ & $\mathbf{1 0 0}$ & $\mathbf{5 2}$ & $\mathbf{1 0 0}$ & $\mathbf{5 8}$ & $\mathbf{1 0 0}$ & $\mathbf{2 6 3}$ & $\mathbf{1 0 0}$ \\
\hline
\end{tabular}

As pesquisas qualitativas direcionam-se a uma experiência social, ancoram-se na fonte oral e se encaminham na busca de significados de vivências para os sujeitos. Dispõem de instrumentos adaptáveis ao processo de cuidar em enfermagem e, nesse sentido, as questões que emergem das 
vivências do assistir em enfermagem podem ser respondidas fornecendo subsídios, por meio das evidências científicas, para reorientar as ações profissionais e nortear as práticas assistenciais.

No conjunto dos trabalhos analisados, observouse $o$ interesse do estudante em conhecer aspectos subjetivos da assistência sob a ótica do usuário, dos familiares e da equipe de saúde. Entre as 263 monografias, $124(47,1 \%)$ estão focados nas percepções, significados e reflexões, principalmente do usuário sobre sua doença e/ou assistência, seguidos das percepções da Enfermagem em relação ao cuidar, à atuação dos profissionais e também dos familiares dos usuários, sobre como a doença afetou suas vidas.

A predominância de estudos qualitativos pode ser justificada pelo direcionamento da Enfermagem para o cuidado dos seres humanos, o que requer conhecer as suas percepções, experiências e subjetividades a fim de traçar linhas de ação para o cuidado.

Percebe-se o uso das tecnologias leves, que são tecnologias das relações, como acolhimento, vínculo, automatização, responsabilização e gestão como forma de gerenciar processos de trabalho, que exigem do profissional de saúde, especialmente do enfermeiro, capacidade diferenciada de criatividade, escuta, flexibilidade e sensibilidade ${ }^{(8)}$. Nesta abordagem, o trabalhador de saúde, junto ao usuário, produz e reproduz relações de subjetividade em que a escuta, a fala, a interpretação, a cumplicidade e a confiabilidade produzem vínculos aprendidos nos trabalhos qualitativos ${ }^{(12)}$.
Verificou-se que $55(20,9 \%)$ dos trabalhos enfocam aspectos epidemiológicos, o que contribui para a formação do estudante, tendo a epidemiologia como ferramenta que aponta para um melhor desempenho profissional. Ressalta-se também a necessidade de agregar $O$ instrumental da epidemiologia para descrição das realidades locais. A Tabela 3 mostra, ainda, que $35(13,3 \%)$ dos estudos têm foco na área profissional de enfermagem e têm como objeto de estudo questões profissionais e pessoais, principalmente do enfermeiro, como a autonomia e a relação dos profissionais de enfermagem com os demais.

\section{Tipos de estudos}

Os estudos foram classificados como exploratórios, descritivos e experimentais. Estudos exploratórios são aqueles que permitem ao investigador aumentar sua experiência em torno de um determinado problema. Os descritivos, por sua vez, pretendem descrever com exatidão os fatos ou fenômenos de determinada realidade, incluindo os estudos de casos e os ex-post-facto. Já os estudos experimentais exigem planejamento rigoroso e a definição precisa de hipóteses.

Os resultados mostraram um número expressivo de estudos descritivos - $228(86,7 \%)$, que se caracterizam pela descrição dos fatos ou fenômenos de determinada realidade, seguidos de descritivos e exploratórios, $11(4,1 \%)$ e $2(0,8 \%)$ experimentais (Tabela 4).

Tabela 4: Tipos de estudos utilizados nas monografias do Curso de Graduação em Enfermagem da UNIMONTES no período de 2001 a 2006. Biblioteca Central da UNIMONTES, 2007.

\begin{tabular}{|c|c|c|c|c|c|c|c|c|c|c|c|c|c|c|}
\hline \multirow{2}{*}{$\begin{array}{l}\text { Tipos Anos } \\
\text { de Estudo }\end{array}$} & $\mathbf{2 0 0 1}$ & $\mathbf{2 0 0 2}$ & $\mathbf{2 0 0 3}$ & $\mathbf{2 0 0 4}$ & $\mathbf{2 0 0 5}$ & \multicolumn{2}{|c|}{$\mathbf{2 0 0 6}$} & \multicolumn{2}{|c|}{ Total } \\
\cline { 2 - 17 } & $\mathrm{N}$ & $\%$ & $\mathrm{~N}$ & $\%$ & $\mathrm{~N}$ & $\%$ & $\mathrm{n}$ & $\%$ & $\mathrm{n}$ & $\%$ & $\mathrm{n}$ & $\%$ & $\mathrm{n}$ & $\%$ \\
\hline Descritivo & 34 & 85 & 29 & 85,2 & 37 & 94,9 & 38 & 95 & 43 & 82,7 & 47 & 81 & 228 & 86,7 \\
\hline Exploratório & 3 & 7,5 & 1 & 2,9 & 2 & 5,1 & - & - & 4 & 7,7 & - & - & 10 & 3,8 \\
\hline Descritivo e Exploratório & 2 & 5 & - & - & - & - & - & - & 2 & 3,8 & 7 & 12 & 11 & 4,1 \\
\hline Experimental & - & - & - & - & - & - & - & - & - & - & 2 & 3,5 & 2 & 0,8 \\
\hline Observacional & - & - & - & - & - & - & - & - & - & - & 2 & 3,5 & 2 & 0,8 \\
\hline $\begin{array}{c}\text { Não especificado } \\
\text { adequadamente }\end{array}$ & 1 & 2,5 & 4 & 11,9 & - & - & 2 & 5 & 3 & 5,8 & - & - & 10 & 3,8 \\
\hline TOTAL & $\mathbf{4 0}$ & $\mathbf{1 0 0}$ & $\mathbf{3 4}$ & $\mathbf{1 0 0}$ & $\mathbf{3 9}$ & $\mathbf{1 0 0}$ & $\mathbf{4 0}$ & - & $\mathbf{5 2}$ & $\mathbf{1 0 0}$ & $\mathbf{5 8}$ & $\mathbf{1 0 0}$ & $\mathbf{2 6 3}$ & $\mathbf{1 0 0}$ \\
\hline
\end{tabular}

Esses achados podem certamente serem explicados pela preferência dos estudantes de graduação por estudos menos complexos e com abordagens iniciais de aproximação.

Os estudos descritivos facilitam a inserção do estudante e a viabilidade da pesquisa em tempo compatível ao término da graduação(16). 0 mesmo ocorre com os graduandos do 8o período do Curso de Graduação da UNIMONTES, visto que se dedicam à realização do TCC nos períodos finais da graduação, quando estão sobrecarregados, o que impossibilita a realização de estudos que demandem mais tempo de realização como os estudos prospectivos.

Uma recomendação é que o TCC seja introduzido no início do Curso, por meio da Iniciação Científica. Assim, a pesquisa será uma trajetória na vida do acadêmico e não um trabalho final. 
Também os docentes devem ser melhor capacitados para estudos quantitativos, uma vez que não se pode negar a importância de quantificar e medir fenômenos que ocorrem no campo da Saúde. Os estudos quantitativos permitem avaliar a importância, a gravidade, o risco, e a tendência de agravos e ameaças. Tratam de probabilidades, associações estatisticamente significantes para se conhecer uma realidade. Ademais, os métodos quantitativos são aptos a dar sólidos fundamentos às descobertas e às hipóteses formuladas, sendo orientados para a busca da magnitude e das causas dos fenômenos sociais, sem interesse pela dimensão subjetiva e utilizando procedimentos controlados ${ }^{(18)}$.

Acrescenta-se que muitas inquietações que surgem da prática profissional do enfermeiro não podem ser respondidas pelos estudos experimentais. Por isso busca-se o referencial metodológico qualitativo, como também foi reconhecido neste estudo, para se compreenderem questões relacionadas à existência humana, extraídas do cotidiano profissional, que não podem ser discutidas apenas como fatos passíveis de causalidade, repetição e controle ${ }^{(5)}$.

\section{I nstrumentos utilizados}

Em relação aos tipos de instrumentos utilizados para a coleta de dados, a entrevista foi a priorizada em 148 (53\%) das monografias. Em seguida, aparece o formulário, $58 \quad(20,8 \%)$, que é caracterizado pelo contato face a face entre o entrevistador e o informante durante a coleta de dados, e o questionário, 49 (17,5\%), que objetiva descrever as características e medir determinadas variáveis, devendo ser respondido por escrito e sem a presença do entrevistador, conforme mostra a Tabela 5.

Tabela 5: Tipos de instrumentos de coleta de dados utilizados nas monografias do Curso de Graduação em Enfermagem da UNIMONTES no período de 2001 a 2006. Biblioteca Central da UNIMONTES, 2007.

\begin{tabular}{|c|c|c|c|c|c|c|c|c|c|c|c|c|c|c|}
\hline \multirow{2}{*}{$\begin{array}{|ll|}\text { Tipos de instrumentos } & \text { Anos } \\
\end{array}$} & \multicolumn{2}{|c|}{2001} & \multicolumn{2}{|c|}{2002} & \multicolumn{2}{|c|}{2003} & \multicolumn{2}{|c|}{2004} & \multicolumn{2}{|c|}{2005} & \multicolumn{2}{|c|}{2006} & \multicolumn{2}{|c|}{ Total } \\
\hline & $\mathbf{n}$ & $\%$ & $\mathbf{n}$ & $\%$ & $\mathbf{n}$ & $\%$ & $\mathbf{n}$ & $\%$ & $\mathbf{n}$ & $\%$ & $\mathbf{n}$ & $\%$ & $\mathbf{n}$ & $\%$ \\
\hline Entrevista & 19 & 37,3 & 15 & 44,1 & 24 & 60 & 23 & 53,4 & 37 & 69,8 & 30 & 51,7 & 148 & 53 \\
\hline Que & 10 & 19,6 & 5 & 14,7 & 4 & 10 & 10 & 23,3 & 8 & 15,1 & 12 & 20,7 & 49 & 17,5 \\
\hline & 17 & 33,3 & 8 & 23,5 & 8 & 20 & 7 & 16,3 & 6 & 11,3 & 12 & 20,7 & 58 & 20,8 \\
\hline $\mathrm{G}$ & 2 & 3,9 & - & - & 2 & 5 & 2 & 4,7 & 2 & 3,8 & 2 & 3,5 & 10 & 3,6 \\
\hline Mal & 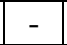 & - & 2 & 5,9 & 2 & J & 1 & 2,3 & - & 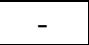 & 1 & 1,7 & 6 & 2,2 \\
\hline Não especificado adequad & 3 & 5,9 & 4 & 11,8 & - & - & - & - & - & - & 1 & 1,7 & 8 & 2,9 \\
\hline TOTAL & 51 & 100 & 34 & 100 & 40 & 100 & 43 & 100 & 53 & 100 & 58 & 100 & 279 & 100 \\
\hline
\end{tabular}

A entrevista é amplamente utilizada como instrumento na assistência e na pesquisa em Enfermagem e se mostra como técnica adequada à obtenção de dados. Revela informações sobre assuntos complexos e verifica sentimentos subjacentes à determinada questão apresentada, sendo, portanto, o instrumento adequado em estudos qualitativos, ficando justificada a sua maior escolha pelos acadêmicos em seus estudos.

Vale ressaltar que $16(6,1 \%)$ dos pesquisadores utilizaram mais de um instrumento de coleta de dados. Tal fato pode ser atribuído à técnica de triangulação na coleta de dados, que tem como objetivo básico alcançar a máxima amplitude na descrição, explicação e compreensão do foco em estudo, partindo de princípios que sustentam a impossibilidade de conceber a existência isolada de um fenômeno social, sem significados culturais, sem raízes históricas, sem vinculações estreitas e essenciais com uma macrorrealidade social ${ }^{(19)}$.

Esse tipo de abordagem pode ser compreendido também como uma dinâmica de investigação que integra a análise das estruturas, dos processos e dos resultados, a compreensão das relações envolvidas na implementação das ações e a visão que os atores diferenciados constroem sobre todo o projeto, seu desenvolvimento, as relações hierárquicas e técnicas fazendo um construto específico.

Além da integração objetiva e subjetiva nos processos de pesquisa, essa técnica proposta inclui os atores contactados em campo não como objetos de análise, mas principalmente como sujeitos de auto-avaliação. Desse modo, proporciona meios de compreensão dos dados, de maneira interativa e intersubjetiva, promovendo maior clareza teórica, com o intuito de superar a dicotomia entre quantitativo versus qualitativo, ao compreender a quantidade como um indicador e parte da qualidade dos fenômenos ${ }^{(19)}$.

Assim sendo, é possível a superação dialética sobre o objetivismo puro, em função da riqueza de conhecimento que pode ser associada à valorização do significado e da intencionalidade dos atos, das relações e das estruturas sociais. Ressalta-se, ainda, que a integração ocorre, sobretudo, quando se trata de processar e analisar dados produzidos por vários instrumentos, na perspectiva de várias disciplinas e por motivos epistemológicos ${ }^{(19)}$.

Essa multiplicidade de recursos de instrumentos de coleta de dados permite ampliar a obtenção de 
dados ganhando em qualidade as possibilidades de interpretação ${ }^{(8)}$, o que propicia o compartilhamento de reflexões de áreas diversas refletindo em avanços importantes na área do conhecimento(19).

\section{Sujeitos do estudo}

Verifica-se na Tabela 6 que houve predominância de estudos que tiveram como sujeitos, o adulto e a mulher, abrangendo, respectivamente, $55(22,9 \%)$ e $50(20,7 \%)$, não sendo muito diferente do que foi observado quanto às preferências temáticas (Tabela 2). Há também a presença do profissional enfermeiro como participante em $28(11,6 \%)$ das pesquisas, seguido pela equipe de enfermagem, 26 (10,8\%).

Tabela 6: Distribuição dos sujeitos dos estudos nas monografias do Curso de Graduação em Enfermagem da UNIMONTES no período de 2001 a 2006. Biblioteca Central da UNIMONTES, 2007.

\begin{tabular}{|c|c|c|c|c|c|c|c|c|c|c|c|c|c|c|}
\hline \multirow{2}{*}{$\begin{array}{l}\text { Sujeitos Anos } \\
\text { dosestudos }\end{array}$} & $\mathbf{2 0 0 1}$ & $\mathbf{2 0 0 2}$ & $\mathbf{2 0 0 3}$ & $\mathbf{2 0 0 4}$ & $\mathbf{2 0 0 5}$ & $\mathbf{2 0 0 6}$ & \multicolumn{2}{c|}{ Total } \\
\cline { 2 - 14 } & $\mathbf{n}$ & $\mathbf{\%}$ & $\mathbf{N}$ & $\mathbf{\%}$ & $\mathbf{n}$ & $\mathbf{\%}$ & $\mathbf{n}$ & $\mathbf{\%}$ & $\mathbf{n}$ & $\mathbf{\%}$ & $\mathbf{n}$ & $\mathbf{\%}$ & $\mathbf{N}$ & $\mathbf{\%}$ \\
\hline Acadêmico de Enfermagem & - & - & - & - & 01 & 2,7 & 03 & 7,5 & - & - & 01 & 1,8 & 05 & 2,1 \\
\hline Agente Comunitário de Saúde & 01 & 2,9 & - & - & 01 & 2,7 & - & - & 01 & 2,1 & - & - & 03 & 1,2 \\
\hline Adolescente & 01 & 2,9 & 01 & 3,7 & 02 & 5,4 & 02 & 5 & 02 & 4,2 & 05 & 9,1 & 13 & 5,4 \\
\hline Adulto & 08 & 23,6 & 03 & 11,1 & 11 & 29,8 & 08 & 20 & 10 & 20,8 & 15 & 27,3 & 55 & 22,9 \\
\hline Criança & 01 & 2,9 & 01 & 3,7 & 02 & 5,4 & 02 & 5 & 02 & 4,2 & 03 & 5,5 & 11 & 4,6 \\
\hline Cuidador & - & - & - & - & - & - & 01 & 2,5 & 01 & 2,1 & 03 & 5,5 & 05 & 2,1 \\
\hline Enfermeiro & 02 & 5,9 & 04 & 14,8 & 03 & 8,1 & 02 & 5 & 09 & 18,7 & 08 & 14,5 & 28 & 11,6 \\
\hline Equipe de Enfermagem & - & - & 05 & 18,5 & 06 & 16,2 & 06 & 15 & 04 & 8,3 & 05 & 9,1 & 26 & 10,8 \\
\hline Equipe Multiprofissional & 03 & 8,8 & 01 & 3,7 & 03 & 8,1 & 03 & 7,5 & 02 & 4,2 & 02 & 3,6 & 14 & 5,8 \\
\hline Família & 06 & 17,7 & 01 & 3,7 & 03 & 8,1 & - & - & 03 & 6,2 & 02 & 3,6 & 15 & 6,2 \\
\hline Idoso & 03 & 8,8 & - & - & 01 & 2,7 & 05 & 12,5 & 02 & 4,2 & 03 & 5,5 & 14 & 5,8 \\
\hline Mulher & 07 & 20,6 & 11 & 40,8 & 04 & 10,8 & 08 & 20 & 12 & 25 & 08 & 14,5 & 50 & 20,7 \\
\hline Professor & 02 & 5,9 & - & - & - & - & - & & - & - & - & - & 2 & 0,8 \\
\hline TOTAL & $\mathbf{3 4}$ & $\mathbf{1 0 0}$ & $\mathbf{2 7}$ & $\mathbf{1 0 0}$ & $\mathbf{3 7}$ & $\mathbf{1 0 0}$ & $\mathbf{4 0}$ & $\mathbf{1 0 0}$ & $\mathbf{4 8}$ & $\mathbf{1 0 0}$ & $\mathbf{5 5}$ & $\mathbf{1 0 0}$ & $\mathbf{2 4 1}$ & $\mathbf{1 0 0}$ \\
\hline
\end{tabular}

\section{Cenários das pesquisas}

Verifica-se na Tabela 7 que nas 263 monografias, os cenários de estudo selecionados pelos estudantes para o desenvolvimento das pesquisas foram os Hospitais, 107 (39,7\%), seguidos da Estratégia Saúde da Família (ESF), 92 (34,1\%).
Essa preferência justifica-se certamente pela maior facilidade de acesso, uma vez que esses cenários representam os principais ambientes onde são realizados os ensinos clínicos e parte do estágio curricular supervisionado do Curso de Graduação em Enfermagem.

Tabela 7: Distribuição dos cenários das pesquisas presentes nas monografias do Curso de Graduação em Enfermagem da UNIMONTES no período de 2001 a 2006. Biblioteca Central da UNIMONTES, 2007.

\begin{tabular}{|c|c|c|c|c|c|c|c|c|c|c|c|c|c|c|}
\hline \multirow{2}{*}{$\begin{array}{l}\text { Cenários Anos } \\
\text { das pesquisas }\end{array}$} & $\mathbf{2 0 0 1}$ & $\mathbf{2 0 0 2}$ & $\mathbf{2 0 0 3}$ & $\mathbf{2 0 0 4}$ & $\mathbf{2 0 0 5}$ & $\mathbf{2 0 0 6}$ & \multicolumn{2}{|c|}{ Total } \\
\cline { 2 - 15 } & $\mathbf{n}$ & $\mathbf{\%}$ & $\mathbf{N}$ & $\mathbf{\%}$ & $\mathbf{n}$ & $\mathbf{\%}$ & $\mathbf{n}$ & $\mathbf{\%}$ & $\mathbf{N}$ & $\mathbf{\%}$ & $\mathbf{n}$ & $\mathbf{\%}$ & $\mathbf{n}$ & $\mathbf{\%}$ \\
\hline Asilo & 01 & 2,1 & 01 & 2,9 & - & - & 02 & 5 & - & - & 1 & 1,8 & 5 & 1,8 \\
\hline Escola & 02 & 4,3 & 01 & 2,9 & - & - & 02 & 5 & - & - & 3 & 5,2 & 8 & 3 \\
\hline Fundação Sara & 01 & 2,1 & 01 & 2,9 & 01 & 2,6 & - & - & 01 & 1,9 & - & - & 4 & 1,5 \\
\hline Gerência Regional de Saúde & 02 & 4,3 & - & - & - & - & - & - & 01 & 1,9 & - & - & 3 & 1,1 \\
\hline Hospital & 16 & 34 & 09 & 26,5 & 13 & 33,3 & 19 & 47,5 & 26 & 50 & 24 & 41,4 & 107 & 39,7 \\
\hline IBGE & 01 & 2,1 & - & - & - & - & - & - & - & - & 1 & 1,7 & 2 & 0,7 \\
\hline Policlínica & 03 & 6,4 & 02 & 5,9 & 02 & 5,1 & 03 & 7,5 & 03 & 5,8 & - & - & 13 & 4,8 \\
\hline Programa de Saúde da Família & 13 & 27,7 & 11 & 32,4 & 17 & 43,6 & 10 & 25 & 16 & 30,8 & 25 & 43,2 & 92 & 34,1 \\
\hline Residência & 03 & 6,4 & 02 & 5,9 & - & - & - & - & - & - & 1 & 1,7 & 6 & 2,2 \\
\hline Serviço Social do Comércio & 01 & 2,1 & - & - & - & - & 01 & 2,5 & - & - & - & - & 2 & 0,7 \\
\hline Secretaria Municipal de Saúde & 01 & 2,1 & 05 & 14,8 & 02 & 5,1 & - & - & 01 & 1,9 & 1 & 1,7 & 10 & 3,7 \\
\hline UNI MONTES & 03 & 6,4 & 01 & 2,9 & 04 & 10,3 & 03 & 7,5 & 03 & 5,8 & 1 & 1,7 & 15 & 5,6 \\
\hline Via Pública & - & - & 01 & 2,9 & - & - & - & - & 01 & 1,9 & 1 & 1,7 & 3 & 1,1 \\
\hline Total & $\mathbf{4 7}$ & $\mathbf{1 0 0}$ & $\mathbf{3 4}$ & $\mathbf{1 0 0}$ & $\mathbf{3 9}$ & $\mathbf{1 0 0}$ & $\mathbf{4 0}$ & $\mathbf{1 0 0}$ & $\mathbf{5 2}$ & $\mathbf{1 0 0}$ & $\mathbf{5 8}$ & $\mathbf{1 0 0}$ & $\mathbf{2 7 0}$ & $\mathbf{1 0 0}$ \\
\hline
\end{tabular}


Além disso, o Projeto Pedagógico do Curso ainda privilegia o cenário hospitalar como principal campo de estágio, o que pode estar associado ao fato de ter sido elaborado há 12 anos. Desse modo, os pressupostos contidos nas Diretrizes Curriculares Nacionais para a área da Enfermagem orientam incluir no currículo a realização de práticas nos mais diversos cenários do cuidar: asilos, creches, domicílios, escolas, orfanatos, enfim, onde a vida acontece e que não se encontram presentes no cotidiano do curso em estudo. Mas vale ressaltar que o PPC encontra-se em fase de reformulação por equipe homologada pela chefia do Departamento de Enfermagem.

Tal fato também pode ser associado à influência direta da proposta flexneriana na constituição das Escolas de Enfermagem no Brasil, priorizando o ensino e a prática hospitalares. Percebe-se que o modelo biomédico encontra-se ainda presente nos estudos desenvolvidos pelos acadêmicos e a existência de uma cultura ainda voltada para os cuidados curativos, hospitalocêntrico, orientando a prática de Enfermagem, torna a produção do conhecimento distante do interesse pela Atenção Primária à Saúde ${ }^{(17)}$.

Também a manutenção das investigações nas instituições hospitalares reflete em parte a herança do modelo hegemônico de assistência à saúde e da formação de recursos humanos para a área, centrada na doença e na cura e conseqüentemente na valorização desses serviços como provedores de pesquisa e de assistência. Contudo, há uma nítida tendência avaliada como positiva para ampliação dos contextos sociais em que a enfermagem atua e uma mudança paradigmática, reflexos dos movimentos sociais, sugerindo uma mudança de concepção do conceito de saúde ${ }^{(6)}$.

Há a necessidade de que essa visão seja ultrapassada para a maior compreensão do processo saúde-doença no atual estágio de desenvolvimento do Sistema Único de Saúde (SUS), tornando-se imprescindível o desenvolvimento de mais pesquisas empíricas e conceituais em cenários diversificados.

A Estratégia Saúde da Família também foi uma opção significativa de cenário para os graduandos, sendo esse um importante cenário para a utilização das tecnologias leves pelos enfermeiros ${ }^{(12)}$.

Deve-se salientar a necessidade da utilização permanente de mecanismos de ajustes da organização dos cenários de aprendizagem quando se busca estudar "a" e "na" realidade. A diversificação de cenários de ensino-aprendizagem constitui-se em uma estratégia propulsora de mudanças profundas no processo de formação profissional. Dessa forma, as experiências mais avançadas nesse terreno têm o propósito de não transformar o espaço dos serviços de Saúde e da comunidade em prolongamentos do hospital universitário e dos centros de saúde-escola. Ou seja, não se pretende descaracterizar os cenários reais como locais de produção de serviços nem transformá-los em locais privilegiados de práticas educacionais $^{(20)}$ e sim em locais de produção e aplicação de conhecimento.

Além disso, a participação de novos sujeitos nesses cenários mostra-se a maneira mais eficaz e verdadeira de trazer novos temas e desafios ao processo de produção do conhecimento e do ensinoaprendizagem.

Verifica-se que foram realizadas $8 \quad(4,02 \%)$ monografias cujos cenários não foram especificados e em $14(7,03 \%)$ foram utilizados dois ou mais cenários (Tabela 7).

\section{CONCLUSÕES}

A partir do exposto, conclui-se que a área assistencial foi a mais contemplada na elaboração das monografias, tendo como principal temática as questões relativas à Saúde da Mulher. Em sua maioria, constituíram-se em estudos de abordagem qualitativa. A coleta de dados foi realizada, principalmente, em Hospitais e na Estratégia de Saúde da Família, sendo a entrevista o instrumento mais utilizado. Os sujeitos eleitos pelos pesquisadores foram a mulher e o adulto.

Adverte-se para a necessidade de realização de pesquisas com temáticas ainda pouco contempladas como ensino, organização dos serviços, novas modalidades de assistência como o processo de trabalho em saúde, sistematização da assistência, atenção e internação domiciliar, entre outras.

Nota-se a ausência de estudos prospectivos e, para tanto, há necessidade de incentivos e investimentos na capacitação dos orientadores e dos preceptores de campo, requerendo, ainda, o contínuo aperfeiçoamento dos mesmos e dos estudantes quanto à condução da pesquisa.

Recomenda-se a realização de outros estudos sobre esta temática e que as encontradas sejam agregadas às preconizadas pelo Grupo de Pesquisa em Enfermagem da UNIMONTES para melhor alicerçar o desenvolvimento de pesquisas na Graduação e na Pós-Graduação.

Sugere-se a inserção precoce dos estudantes em programas de Iniciação Científica, apontada como favorecedora de noções técnicas e metodológicas de pesquisa, buscando estimular o envolvimento de novos pesquisadores na atividade de formação por meio da pesquisa científica. Para tanto, recomendase que o TCC seja introduzido no início do Curso, de forma que a pesquisa seja uma trajetória na vida do acadêmico e não um trabalho final, favorecendo sua formação na pesquisa e na elaboração do TCC, enquanto canal adequado para o desenvolvimento das habilidades e competências. 
Necessita-se de maior diversificação dos sujeitos e dos cenários das investigações, a fim de se contemplarem os diferentes campos da pesquisa em Saúde para se preencherem os inúmeros vazios observados na produção científica dos graduandos de Enfermagem.

Por fim, ressalta-se o caráter pioneiro e pontual do presente estudo, aconselhando a sua continuidade de modo a permitir identificar as tendências, as facilidades e as dificuldades na elaboração dos Trabalhos de Conclusão de Curso para que a atividade de pesquisa do Curso de Enfermagem da UNIMONTES esteja voltada para os preceitos do Sistema Único de Saúde e para as necessidades e demandas de saúde da população, especialmente, da região norte do Estado de Minas Gerais.

\section{REFERÊNCI AS}

1. Giacchero KG, Miasso Al. A produção científica na graduação em enfermagem (1997 a 2004): análise crítica. Rev. Eletr. Enf. [Internet]. 2006 [cited 2009 feb 16];8(3):431-40. Available from: http://www.fen.ufg.br/revista/revista8_3/pdf/v8n3a14.pdf. 2. Saupe R, Wendhausen ALP, Machado HB. Modelo para implantação ou revitalização de trabalhos de conclusão de curso. Rev Latino-am Enfermagem [Internet]. 2004 [cited 2009 feb 16]; 12(1): 109-14. Available from: http://www. scielo.br/pdf/rlae/v12n1/v12n1a15.pdf.

3. Baggio MA. O Significado de cuidado para profissionais da equipe de enfermagem. Rev. Eletr. Enf. [Internet]. 2006 [cited 2009 feb 16]; 8(3):9-16. Available

from: http://www.fen.ufg.br/revista/revista8_1/original_01.htm.

4. Mantovani MF, Labronici LM, Leão TA, Ribeiro ACS. As diferentes abordagens dos trabalhos de conclusão de curso de graduação em enfermagem da Universidade Federal do Paraná. Rev. Eletr. Enf. [Internet]. 2004 [cited 2009 feb 16];6(3):374-7. Available from: http://www.fen.ufg.br/revista/revista6_3/pdf/08_Ori gnal.pdf.

5. Merighi MAB, Kimura AF. Enfocando estratégias de ensino de pesquisa qualitativa utilizada no curso de graduação da escola de enfermagem da universidade de São Paulo. REME: Rev. Min. Enferm. 2004;8(3): 398-401.

6. Souto CMRM, Pessoa SMF, Damasceno MMC, Araújo TL. Tendências das pesquisas de enfermagem em saúde da mulher no período de 2001 a 2005. Texto contexto-enferm. [Internet]. 2007 [cited 2009 feb 16];16(4):719-26. Available from: http://www. scielo.br/pdf/tce/v16n4/a17v16n4.pdf 7. Pinho IC, Siqueira JCBA, Pinho LMO. As percepções do enfermeiro acerca da integralidade da assistência. Rev. Eletr. Enf. [Internet]. 2006 [cited 2009 feb
16]; 8(1):42-51.

Available

from: http://www.fen.ufg.br/revista/revista8_1/pdf/v8nla06. pdf. 8. Rossi FR, Lima MADS. Acolhimento: tecnologia leve nos processos gerenciais do enfermeiro. Rev Bras Enferm. [Internet]. 2005;58(3):305-10. Available from: http://www. scielo. br/pdf/reben/v58n3/a10v58n3.pdf. 9. Secretaria de Ciência, Tecnologia e Insumos Estratégicos; Ministério da Saúde. Por que pesquisa em saúde? Série B. Textos Básicos de Saúde. Série Pesquisa para Saúde: Textos para Tomada de Decisão [Internet]. Brasília: Ministério da Saúde; 2007 [cited 2009 Mar 09]. Available from: http://portal.saude.gov.br/portal/arquivos/pdf/pq_pe squisa_em saude. pdf.

10. Tyrrel MAR, Cabral IE. A produção científica de enfermagem em saúde da mulher e da criança: panorama brasileiro. Rev. enferm. UERJ. 2005; 13(1): 103-11.

11. Consolidação das Propostas de Linhas de Pesquisa em Enfermagem. In: Anais do 11은 Seminário Nacional de Pesquisa em Enfermagem [Internet]; 2001 mai 27-30; Bélem, São Paulo, Brasil. 2001 [cited 2009 mar 09]. Available from: http://www.abennacional.org. br/download/linhapesq 11senpe.doc.

12. Mehry EE A perda da dimensão cuidadora na produção da saúde - uma discussão do modelo assistencial e da intervenção no seu modo de trabalhar a assistência. In: Campos CR, Malta DC, Reis AT, Santos AD, Merhy EE. Sistema Único de Saúde em Belo Horizonte. Reescrevendo o público. São Paulo: Xamã, 1998. p. 103-120.

13. Alves LMM, Nogueira MS, Godoy S, Cárnio EC. Pesquisa básica na enfermagem. Rev Latino-am Enfermagem. 2004; 12(1): 122-7.

14. Conselho Federal de Enfermagem. Resolução no 290/2004. Fixa as Especialidades de Enfermagem. Rio de Janeiro (Brasil) COFEN; 2004.

15. Erdmann AL, Schlindwein BH, Sousa FGM. A produção do conhecimento: diálogo entre os diferentes saberes. Rev Bras Enferm [Internet]. 2006 [cited 2009 feb 16];59(4):560-4. Available from: http://www.scielo. br/pdf/reben/v59n4/a17v59n4.pdf. 16. Gomes MMF, Sanna MC. A Pesquisa em Enfermagem no congresso de iniciação Cientifica de uma Universidade de São Paulo. Rev Bras Enferm. 2004; 57(5): 574-8.

17. Costa MS, Silva MJ. Tendências da temática qualidade de vida na produção científica de enfermagem. Rev Bras Enferm. 2004; 57(2):208-11.

18. Serapioni M. Métodos qualitativos e quantitativos na pesquisa social em saúde: algumas estratégias para a integração. Ciênc. saúde coletiva. [Internet]. 2000;5(1):187-92. Available from: http://www.scielo.br/pdf/csc/v5n1/7089.pdf. 
19. Minayo MCS. O desafio do conhecimento: pesquisa qualitativa em saúde. São Paulo: Hucitec; 2006.

20. Feuerwerker L, Costa $\mathrm{H}$, Rangel ML. Diversificação de cenários de ensino e trabalho sobre necessidades/problemas da comunidade. Divulgação em Saúde para Debate. 2000; (22):36-48.

Artigo recebido em 12.12.07.

Aprovado para publicação em 31.03.09. 